\title{
SYSTEMATIC DEVELOPMENT OF A FAMILY OF FAIR EXCHANGE PROTOCOLS
}

\author{
Paul D Ezhilchelvan and Santosh K Shrivastava \\ University of Newcastle, Newcastle upon Tyne, NE1 7RU, UK
}

\begin{abstract}
Exchange protocols play an important role in application areas such as ecommerce where protocol participants require mutual guarantees that a transaction involving exchange of items has taken place in a specific manner. A protocol is fair if no protocol participant can gain any advantage over an honest participant by misbehaving. This paper presents a family of fair exchange protocols for two participants under a variety of assumptions concerning participant misbehaviour, node reliability and message delays.
\end{abstract}

Keywords: Fair Exchange, Security, Digital Signatures, Crash tolerance, Distributed Systems, Trusted Third Party.

\section{INTRODUCTION}

Fair exchange protocols play an important role in application areas where protocol participants require mutual guarantees that an exchange of data items has taken place in a specific manner. An exchange is fair if a dishonest participant cannot gain any advantage over honest participants by misbehaving. Two-participant fair-exchange protocols that make use of a trusted third party have been studied extensively in the literature (e.g., [1, 5, 11 ]); these protocols maintain fairness even if the dishonest participant can tamper with the protocol execution in an unrestricted (malicious) manner. They however require that an honest participant's node execute the protocol correctly - suffering no failures. We call them non-fault-tolerant. A crashtolerant fair exchange protocol, on the other hand, ensures no loss of fairness to an honest participant even if the participant's node crashes during the execution, provided it eventually recovers [4].

In this paper we develop a number of fair exchange protocols under a variety of assumptions concerning i) user misbehaviour, ii) node failures and iii) communication delays. The development is systematic: we begin by classifying dishonest participants into restricted abusers (they cannot tamper 
with the protocol execution in an arbitrary manner) and unrestricted or malicious abusers, and the communication model into synchronous, where a known bound on message delays exists, and asynchronous; we develop the first protocol under the most constrained set of assumptions: restricted abuser, synchronous communication and no fault tolerance. We then relax the restricted abuser assumption to malicious abuser and then the synchrony assumption into asynchrony. The resulting family of non-fault-tolerant protocols is then transformed into a family of crash-tolerant ones.

A major advantage of our approach is that it enables a reader to gain a deeper understanding of the impact of a given set of assumptions on the problem of fair exchange; it also highlights the relationships that exist between fairness, fault tolerance and communication delay assumptions. We first describe the problem and the underlying system models (section 2), and then develop a family of non-fault-tolerant protocols (section 3), followed by their crash-tolerant counterparts (section 4). Section 5 summarises the characteristics of the protocols developed and concludes the paper.

\section{SYSTEM MODELS AND THE PROBLEM DESCRIPTION}

We consider two mutually untrusting users, $U_{A}$ and $U_{B}$, who have data items $I_{A}$ and $I_{B}$ respectively that are unknown to the other user prior to the exchange. User $U_{x}, X \in\{A, B\}$, intends to send $I_{x}$ in return for receiving $I_{Y}$, where $Y \in\{A, B\}$ and $Y \neq X . P_{x}$ denotes the process that executes an exchange protocol on behalf of user $\mathrm{U}_{\mathbf{x}}$ on his node $\mathrm{N}_{\mathbf{x}}$.

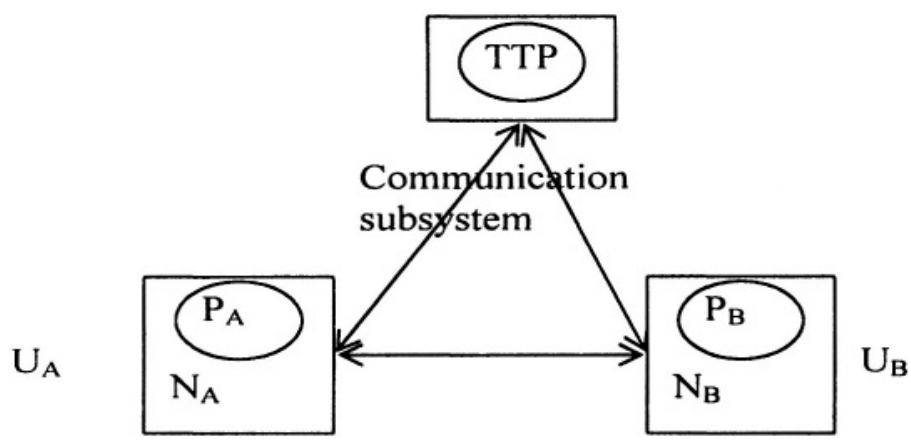

Figure 1. The Fair-Exchange System.

Our distributed exchange system (Figure 1) has a third node hosting the trusted third party (TTP) process. The exchange must preserve fairness as 
well as non-repudiation: a user gets irrefutable evidence of the actions performed by his process (to guard against the other user's false denial of the occurrence of an action or event).

\subsection{Classifying User Misbehaviour}

Generally, the problem of fair exchange is addressed in a context where a dishonest user, say $U_{B}$, totally controls the behaviour of $P_{B}$ and tries to undermine every attempt to ensure fairness and non-repudiation. We term those dishonest users as malicious abusers and distinguish such users from a class of restricted abusers: a dishonest user is said to be a restricted abuser if he is unable to obtain the values of variables or procedures contained in a piece of (binary) code which his local process receives from the TTP and uses during protocol execution. Say, user $U_{B}$ is a restricted abuser and the TTP gives $\mathbf{P}_{\mathbf{B}}$ a piece of code $\Pi_{\mathbf{B}}$ that contains, say, some secret encryption keys. By ourdefinition, $U_{B}$ cannot obtain the keys in $\Pi_{B}$ by visual inspection or through a trace analysis even after $P_{B}$ has executed $U_{B}$. The definition does not however restrict the ability of $U_{B}$ to delay, block, inspect, or tamper with any message which an execution of $\Pi_{B}$ generates or is destined to receive.

One way to realise our notion of a restricted abuser is to have each $\mathbf{P}_{\mathbf{X}}$ receive the TTP code $\Pi_{\mathbf{x}}$ via a secure channel and execute it in a tamperproof part of the user node. We note here that there is much interest in developing tamper-proof computing subsystems by the industry led Trusted Computing Platform Alliance, TCPA [8]. Further, smartcards can be used to achieve such tamper-proof, computing subsystems for small, critical computations $[2,10]$. Thus, developing fair-exchange protocols under the restricted abuser model is of practical interest, and also exposes the benefits that the model has to offer.

\subsection{Classifying Node Behaviour}

A fair-exchange protocol is fault-tolerant if it ensures that fairness is preserved to an honest participant despite the participant's node suffers failures of the assumed type. In other words, an honest user does not suffer loss of fairness when his node is unreliable. We consider two types of node behaviour:

Reliable: An honest user's node never fails.

Crash-recovery: An honest user's node fails by stopping to function (crash); it recovers within some finite (but unknown) time after a crash and 
may crash again after its recovery. An honest user's node has access to a stable store whose contents survive the node crash.

When a user node is regarded to be reliable, the cause of any node crash is attributed to the user who has misbehaved and is therefore not entitled to any fairness guarantee. This is the traditional view taken in the TTP-based protocols (e.g., [1, 5, 11]) which are therefore not fault-tolerant (see [4]). In line with the existing, non-fault-tolerant protocols, the TTP is assumed to be reliable and secure against intrusions and Trojan horses.

\subsection{Classifying Communication Delays}

Communication between correctly functioning processes is assumed to be resilient to network failures: a message loss is tolerated by retransmission and a message corruption is detected using encryption and reduced to a message loss. A network intruder is therefore assumed to be a restricted abuser: a message in transit is a black box to him; he cannot modify it and have it undetected at the destination. He can only delay a message from being accepted at a destination. We consider two types of network intrusion: intruder gives up delaying a given message transmission after a known period of time or after some unknown finite time, leading to two models: in the synchronous model, correct processes exchange messages with delays bounded by a known $D$; in the asynchronous model, $D$ is finite but unknown.

\subsection{Properties of a fair exchange Protocol}

A user is honest if he makes no attempt to modify the behaviour of a protocol process except through the permitted operations.

Termination: Any execution of the protocol terminates for an honest user $U_{X}$. Termination for an honest $U_{X}$ can be either a normal termination in which $P_{X}$ delivers $I_{Y}$ to $U_{X}$ or an exceptional termination where $P_{X}$ informs $\mathrm{U}_{\mathrm{X}}$ that the exchange is unsuccessful.

No_Loss_Of_Fairness: If $\mathrm{P}_{\mathrm{X}}$ of honest $\mathrm{U}_{\mathrm{X}}$ terminates exceptionally, $\mathrm{U}_{\mathrm{Y}}$ does not receive $\mathbf{I}_{\mathbf{X}}$.

Non-repudiation: when $\mathrm{P}_{\mathbf{X}}$ delivers $\mathrm{I}_{\mathbf{Y}}$ to honest $\mathbf{U}_{\mathbf{X}}$, it also provides irrefutable evidence against any false denial on $I_{Y}$ having been sent by $U_{Y}$.

The above properties are met trivially if $P_{A}$ and $P_{B}$ terminate always exceptionally.

Non-Triviality: If $\mathrm{U}_{\mathrm{A}}$ and $\mathrm{U}_{\mathrm{B}}$ are honest, both are guaranteed to have normal termination.

Non-triviality is easy to guarantee for the synchronous, non-fault-tolerant case. When the fair-exchange protocol is deterministic (not probabilistic) in 
nature, honesty of both the users alone is not sufficient to guarantee nontriviality for the case of crash-recovery nodes and synchronous communication, as well for the case of asynchronous communication. The reasons for this inability are as follows. In crash-recovery model, the bound on the time taken by an honest user node to recover from a crash is unknown and a dishonest user may never allow his node to recover. So, a correctly functioning process (of an honest user or the TTP) that is waiting too long for a message from a user process cannot resolve whether the latter is honest and its message is unduly delayed due to a crash or is dishonest and is not going to transmit the expected message; similarly, in the asynchronous model, it cannot resolve whether the user process from whom a message is expected is honest and its message is still in transit or is dishonest and the message will not be transmitted at all. (Readers familiar with the FLP impossibility result [3] may find these arguments remarkably similar.) Therefore, meeting the termination property would mean that an execution of the protocol may have to terminate exceptionally even if both users are honest. Therefore, the necessary condition for ensuring non-triviality is that the honest users be willing to re-execute the protocol after every exceptional termination; the sufficient condition differs with the combination of assumptions and is stated below: there must be an execution in which

(i) user nodes do not crash, for the case of crash-recovery nodes and synchronous communication;

(ii) message delays do not increase, for the case of reliable nodes and asynchronous communication; and,

(i) and (ii), for the case of crash-recovery nodes and asynchronous communication.

\subsection{Assumptions, Notations and the Exchange Preliminaries}

In all our protocols, the TTP, assumed to be secure and reliable, is used to set-up the context for, and to initiate, the actual exchange. We make the following additional assumptions.

A1: Processing within correctly functioning nodes is synchronous: delays for task scheduling and processing are bounded by a known constant.

A2: $\quad$ The clocks of the TTP and the functioning nodes of honest users are synchronised to real-time within a known bound which, for simplicity, is assumed to be zero. A recovering node receives the current time from its user. 


\section{Notations}

M: a message;

$V_{A}\left(V_{B}\right)$ : procedure to verify if $I_{A}\left(I_{B}\right)$ satisfies its description $\Sigma_{A}\left(\Sigma_{B}\right)$ advertised by $\mathrm{U}_{\mathrm{A}}\left(\mathrm{U}_{\mathrm{B}}\right)$.

$\mathrm{eK}(\mathrm{M})$ : encryption of $\mathrm{M}$ using key $\mathrm{K}$;

Sig $(M)$ : signature of $P_{X}, X \in\{A, B\}$, on $M$ using the private key of $U_{X}$;

$\operatorname{Sig}_{\text {TTP }}(M)$ : TTP's signature on $M$. Signatures are both signer- and content-dependent, and are verifiable using the signer's public key.

L: a label that identifies a given exchange;

$\mathrm{N}$ : a large random number (nonce) generated securely by TTP to uniquely identify messages of a given execution of the protocol for a given exchange; and,

$\mathrm{H}$ : one-way and collision-resistant hash function: it is not feasible to compute from $\mathrm{H}(\mathrm{N}), \mathrm{N}$ nor another $\mathrm{N}^{\prime}$ such that $\mathrm{H}(\mathrm{N})=\mathrm{H}\left(\mathrm{N}^{\prime}\right)$. phase.

$\Pi_{\mathrm{x}}$ : contents of a message sent by the TTP to $\mathrm{P}_{\mathrm{x}}$ to initate the exchange

When a party $Z \in\{A, B, T T P\}$ sends a message $M$ it also includes $\operatorname{Sig}_{Z}(M)$ as the evidence of origin for M. A recipient accepts a received $M$ only if the accompanying $\operatorname{Sig}_{z}(\mathbf{M})$ is found authentic. For brevity, we will not make the details of this verification explicit and a received message will only refer to a message received with an authentic evidence of origin; further the pair $\left\{\mathbf{M}, \operatorname{Sig}_{z}(\mathbf{M})\right\}$ will simply be written as $M$ which will be indicated by its significant fields.

\section{Exchange Preliminaries}

All our protocols are structured into two phases: the start-up phase followed by the exchange phase when the actual exchange of promised data items takes place. During the start-up phase, the following three steps are carried out:

Step 0.1: Each $\mathrm{U}_{\mathrm{X}}$ approaches a trusted authority $\mathrm{TA}_{\mathrm{X}}$ to generate a verification procedure $V_{X}$ using which any party can verify if a data item I satisfies specification $\Sigma_{X}$. For example, if $I_{B}$ is a piece of software $S, U_{B}$ must approach a software licensing authority (trusted by $U_{A}$ ) who evaluates $S$ against the specification $\Sigma_{B}$ that $U_{B}$ advertised. If satisfied, the authority computes an evidence of evaluation $E_{B}=H(S) ; V_{B}$ is then generated as a program which contains $E_{B}$ and evaluates the predicate $E_{B}=H(I)$ when invoked to verify a data item I. Using the conventional notations, the step is:

\begin{tabular}{|l|l|}
\hline $\mathrm{U}_{\mathrm{A}} \rightarrow \mathrm{TA}_{\mathrm{A}}:\left(\mathrm{I}_{\mathrm{A}}, \Sigma_{\mathrm{A}}\right) ;$ & $\mathrm{U}_{\mathrm{B}} \rightarrow \mathrm{TA}_{\mathrm{B}}:\left(\mathrm{I}_{\mathrm{B}}, \Sigma_{\mathrm{B}}\right) ;$ \\
$\mathrm{TA}_{\mathrm{A}} \rightarrow \mathrm{U}_{\mathrm{A}}:\left\{\mathrm{V}_{\mathrm{A}}, \Sigma_{\mathrm{A}}, \operatorname{Sig}_{\mathrm{TA}_{-} \mathrm{A}}\left(\mathrm{V}_{\mathrm{A}}\right.\right.$, & $\mathrm{TA}_{\mathrm{B}} \rightarrow \mathrm{U}_{\mathrm{B}}:\left\{\mathrm{V}_{\mathrm{B}}, \Sigma_{\mathrm{B}}, \operatorname{Sig}_{\mathrm{TA}_{-} \mathrm{B}}\left(\mathrm{V}_{\mathrm{B}}\right.\right.$, \\
$\left.\left.\Sigma_{\mathrm{A}}\right)\right\} ;$ & $\left.\left.\Sigma_{\mathrm{B}}\right)\right\} ;$ \\
\hline
\end{tabular}


Step 0.2: Users exchange their $\left\{\mathrm{V}, \Sigma, \operatorname{Sig}_{\mathrm{TA}}(\mathrm{V}, \Sigma)\right\}$, decide on the TTP and on a (future) time $T_{E x}$ when the TTP should initiate the exchange phase.

Step 0.3: $\mathrm{U}_{\mathrm{A}} \rightarrow$ TTP: (A, B, $\left.\mathrm{T}_{\mathrm{EX}},\left\{\mathrm{V}_{\mathrm{A}}, \Sigma_{\mathrm{A}}\right\},\left\{\mathrm{V}_{\mathrm{B}}, \Sigma_{\mathrm{B}}\right\}, \mathrm{rtt}_{\mathrm{AB}}\right)$ : similarly, $\mathrm{U}_{B} \rightarrow$ TTP: $\left(A, B, T_{E X},\left\{V_{A}, \Sigma_{A}\right\},\left\{V_{B}, \Sigma_{B}\right\}, r t t_{B A}\right)$. The last field $r_{t t_{X Y}}$ is $U_{X}$ 's estimation of the round trip time between its node and $N_{Y}$.

Upon receiving messages sent in step 0.3 , the TTP verifies whether all fields except the last one are identical. If so, the TTP decides on a unique L for the exchange if the exchange is new; generates a nonce $\mathrm{N}$ for the exchange phase and records $\mathrm{N}$ in a variable active_run\# $(\mathrm{L})=\mathrm{N}$. If the exchange is not new, it only generates a new nonce and records it in active_run\#(L). It then sends $\Pi_{B}$ to $N_{A}$ and $\Pi_{B}$ to $N_{B}$ which include the parameters $\mathrm{L}$ and $\mathrm{H}(\mathrm{N})$ which are used to identify messages of a given execution. The nature and the complete contents of $\Pi$ 's sent by TTP depend on the exchange phase of the protocol for a chosen combination of various assumptions discussed earlier.

\section{TTP Involvement}

A protocol is said to keep TTP offline if it is possible for honest user processes to achieve normal termination without interacting with TTP after the exchange phase is initiated. Such a protocol is also called optimistic in the literature. If TTP is not off-line, it is said to be on-line.

A protocol is said to use a state-keeping TTP if it requires the TTP to respond to messages from user processes for an unspecified amount of time; the protocol is said to use a state-relinquishing TTP if it requires the TTP only for a specified duration after the exchange phase has been initiated. From cost point of view, TTP is preferred to be offline and staterelinquishing.

\section{NON-FAULT-TOLERANT FAIR EXCHANGE PROTOCOLS}

\subsection{Restricted Abuser, Synchronous Communication (Protocol P1)}

The TTP sends a code $\Pi_{\mathbf{A}}$ to $\mathbf{P}_{\mathbf{A}}$ and $\Pi_{\mathbf{B}}$ to $\mathbf{P}_{\mathbf{B}}$. $\mathbf{P}_{\mathbf{A}}$ and $\mathbf{P}_{\mathbf{B}}$ perform the exchange phase of the protocol P1 by executing the code given to them. Embedded in $\Pi_{\mathbf{X}}$ are $\mathbf{L}, \mathbf{H}(\mathrm{N}), \mathbf{V}_{\mathbf{X}}, \Delta$, and keys $\mathbf{K}_{\mathbf{A}}$ and $\mathbf{K}_{\mathbf{B}}$, each with TTP's evidence of origin, $\Delta$ is set to $D$ (the known bound on message delays), $\mathbf{K}_{A}$ and $\mathbf{K}_{\mathbf{B}}$ are symmetric and random session keys. When a dishonest $\mathbf{U}_{\mathbf{X}}$ is only a restricted abuser, he cannot obtain $\mathbf{K}_{\mathbf{A}}$ and $\mathbf{K}_{\mathbf{B}}$ from $\Pi_{\mathbf{X}}$ nor undetectably modify $\mathbf{V}_{\mathbf{X}}$ embedded within $\Pi_{\mathbf{X}}$. However, $\mathbf{U}_{\mathbf{X}}$ can delay, block, inspect, or 
tamper with any message $P_{x}$ generates or is destined to receive while it executes $\Pi_{X}$.

On executing $\Pi_{A}, P_{A}$ first verifies whether $I_{A}$ (input by $U_{A}$ ) passes $V_{A}$ embedded within $\boldsymbol{I}_{A}$. Only if $I_{A}$ passes the verification, $P_{A}$ continues the execution: it encrypts the verified $I_{A}$ using $K_{A}$, forms a message $\mathbf{M}_{A}$ and encrypts $\mathbf{M}_{\mathbf{A}}$ with $\mathrm{K}_{\mathrm{B}}$. The encrypted $\mathbf{M}_{\mathbf{A}}$, like all messages sent by $\mathbf{P}_{\mathrm{A}}$, contains a signature generated using the private key of $U_{A}$ and is sent to $P_{B}$. (Any message without valid signature is ignored and hence assumed not 'received' by a destination.) $P_{B}$ decrypts the received $M_{A}$, and if the contents are correct, it sends an acknowledgement $\operatorname{Ack}_{B}(A)$ to $P_{A} \cdot P_{B}$ similarly sends $M_{B}$ to, and expects to receive $\operatorname{Ack}_{A}(B)$ from, $P_{A}$. The message exchange between $P_{A}$ and $P_{B}$ are depicted in Figure 2, where a process sending a message shown along an out-going arrow is conditional upon that process having received the messages shown along all incoming arrows. $\mathbf{P}_{\mathbf{X}}$, after receiving $\Pi_{X}$, starts executing both the rounds concurrently: send $\mathbf{M}_{\mathbf{X}}$ and wait for $\operatorname{Ack}_{Y}(X)$ while waiting for $M_{Y}$ before $\operatorname{Ack}_{X}(Y)$ can be sent; a timeout of $2 \Delta$ is used to avoid indefinite waiting.

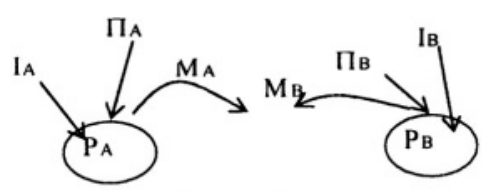

Round I

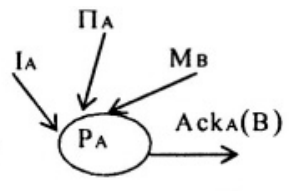

Round 2

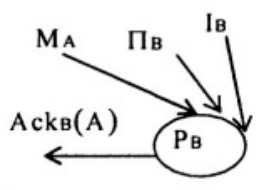

Figure 2. Protocol P1: Message exchange rounds between user processes.

Table 1 summarises the messages used and the intended direction of their flow. A message is indicated by its significant fields, the first three fields will be: the label L, the sender, and $\mathrm{H}(\mathrm{N})$. Note that the first and the third message fields uniquely refer to a given execution.

The condition on $P_{A}\left(P_{B}\right)$ for computing $I_{B}\left(I_{A}\right)$ from $M_{B}\left(M_{A}\right)$ is: both $\mathrm{M}_{B}\left(\mathrm{M}_{\mathrm{A}}\right)$ and $\operatorname{Ack}_{B}(\mathrm{~A})\left(\operatorname{Ack}_{\mathrm{A}}(\mathrm{B})\right)$ must be received within $2 \Delta$ time after receiving $\Pi_{A}\left(\Pi_{B}\right)$ from TTP. If $P_{A}$ receives only $M_{B}$ (within $2 \Delta$ time), it asks TTP to resolve the exchange by sending message $\operatorname{Res}_{A}$ that contains both $\mathbf{M}_{\mathrm{A}}$ and the received $\mathrm{M}_{\mathrm{B}}$. If it receives neither $\mathrm{M}_{\mathrm{B}}$ nor $\operatorname{Ack}_{\mathrm{B}}(\mathrm{A})$, it requests TTP to abort the exchange by sending message $R e q_{A}$ that contains only $\mathbf{M}_{A}$. TTP, having initiated the exchange at its clock time $T_{E X}$, executes the following steps at $\mathrm{T}_{\mathrm{EX}}+4 \Delta$ :

Step T1: if it has received Req from both processes or Res from at least one process, it sets variable outcome = resolved and resolves the exchange by sending $\mathbf{M}_{\mathrm{TTP}}(\mathrm{X})$ to both processes; 
Step T2: $\quad$ if it has received Req from only one process, it sets outcome $=$ aborted and aborts the exchange by sending $\operatorname{Abort}_{\mathrm{TTP}}(\mathrm{X})$ to both processes;

Step T3: if it has received no message, it sets variable outcome to unknown;

Step T4: it terminates (as a state-relinquishing TTP).

\begin{tabular}{|c|c|}
\hline $\mathrm{K}_{\mathrm{A}}\left(\mathrm{I}_{\mathrm{A}}\right)$ & в) \\
\hline $\mathrm{M}_{\mathrm{A}}=\mathrm{eK}_{\mathrm{B}}(\mathrm{L}, \mathrm{A}, \mathrm{H}(\mathrm{N}$ & $\mathrm{M}_{\mathrm{B}}=\mathrm{eK}_{\mathrm{A}}(\mathrm{L}, \mathrm{B}, \mathrm{H}(\mathrm{N})$, \\
\hline $\begin{array}{l}=\left(\mathrm{L}, \mathrm{A}, \mathrm{H}(\mathrm{N}), \mathrm{H}\left(\mathrm{M}_{\mathrm{B}}\right)\right. \\
\mathrm{A} \rightarrow \mathrm{B}\end{array}$ & $\mathrm{H}\left(\mathrm{M}_{\mathrm{A}}\right)$ \\
\hline $\begin{array}{l}\operatorname{Res}_{\mathrm{A}}=\left(\mathrm{L}, \mathrm{A}, \mathrm{H}(\mathrm{N}), \mathrm{M}_{\mathrm{A}}, \mathrm{M}_{\mathrm{B}},\right. \\
\text { Resolve_request }) ; \mathrm{A} \rightarrow \mathrm{TTP}\end{array}$ & $\begin{array}{l}\operatorname{Res}_{\mathrm{B}}=\left(\mathrm{L}, \mathrm{B}, \mathrm{H}(\mathrm{N}), \mathrm{M}_{\mathrm{B}}, \mathrm{M}_{\mathrm{A}},\right. \\
\text { Resolve_request }) ; \mathrm{B} \rightarrow \mathrm{TTP}\end{array}$ \\
\hline $\begin{array}{l}\operatorname{Req}_{\mathrm{A}}=\left(\mathrm{L}, \quad \mathrm{A}, \quad \mathrm{H}(\mathrm{N}), \quad \mathrm{M}_{\mathrm{A}},\right. \\
\text { Abort_request }) ; \mathrm{A} \rightarrow \mathrm{TTP}\end{array}$ & $\begin{array}{l}\operatorname{Req}_{\mathrm{B}}=\left(\mathrm{L}, \mathrm{B}, \mathrm{H}(\mathrm{N}), \quad \mathrm{M}_{\mathrm{B}},\right. \\
\text { Abort_request }) ; \mathrm{B} \rightarrow \text { TTP }\end{array}$ \\
\hline $\begin{array}{l}\mathrm{A})=\left(\mathrm{L}, \mathrm{TTP}, \mathrm{H}(\mathrm{N}), \mathrm{M}_{\mathrm{B}},\right. \\
c k) ; \mathrm{TTP} \rightarrow \mathrm{A}\end{array}$ & $\begin{array}{l}\mathrm{P}(\mathrm{B})=\left(\mathrm{L}, \mathrm{TTP}, \mathrm{H}(\mathrm{N}), \mathrm{M}_{\mathrm{A}},\right. \\
\text { ack); TTP } \rightarrow \mathrm{B}\end{array}$ \\
\hline $\begin{array}{l}(\mathrm{A})=(\mathrm{L}, \quad \mathrm{TTP}, \\
\text { granted, } \mathrm{A}) ; \mathrm{TTP} \rightarrow \mathrm{A}\end{array}$ & $\begin{array}{l}\text { Abort }_{\mathrm{TTP}}(\mathrm{B})=(\mathrm{L}, \mathrm{TTP}, \mathrm{H}( \\
\text { Abort_granted, B); TTP } \rightarrow \mathrm{B}\end{array}$ \\
\hline
\end{tabular}

Table 1. Description of messages used in Protocol P1.

An honest $\mathbf{P}_{\mathbf{A}}$ terminates normally by receiving either $\mathbf{M}_{\mathrm{B}}$ and $\operatorname{Ack}_{\mathrm{B}}(\mathrm{A})$ or $\mathbf{M}_{\mathrm{TTP}}(\mathrm{A})$; exceptionally by receiving $\mathrm{Abort}_{\mathrm{TTP}}(\mathrm{A})$. The protocol keeps TTP off-line (step T3 above). The four properties of fair-exchange (Section 2.4) are met by P1 because: (i) dishonest user, say $\mathrm{U}_{\mathrm{B}}$, is a restricted abuser, so he cannot force $\mathbf{P}_{\mathbf{B}}$ to deliver $\mathbf{I}_{\mathbf{A}}$ against the protocol conditions nor can he obtain $\mathbf{K}_{\mathbf{A}}$ and $\mathbf{K}_{\mathbf{B}}$ from $\Pi_{\mathbf{B}}$; (ii) an honest $\mathbf{P}_{\mathbf{A}}$ sends $\mathbf{A c k}_{\mathbf{A}}(\mathbf{B})$ to $\mathbf{P}_{\mathbf{B}}$ only if it receives $\mathbf{M}_{\mathbf{B}}$ in a timely manner; (iii) any message that $\mathbf{P}_{\mathbf{A}}$ sends to TTP reaches before $T_{E X}+4 \Delta$; and, (iv) TTP's response, if any, is identical to both $\mathbf{P}_{\mathrm{A}}$ and $\mathbf{P}_{\mathrm{B}}$.

\subsection{Malicious Abuser, Synchronous Communication (P1.1)}

1. P1.1 is the same as P1 except some messages of the exchange phase have different contents. This is because a dishonest user, say $\mathbf{U}_{B}$, can obtain the keys $\mathbf{K}_{\mathbf{A}}$ and $\mathrm{K}_{\mathrm{B}}$ in the program $\Pi_{\mathbf{B}}$ supplied by TTP at the start of the exchange phase. The following changes are needed: 
2. $\Pi_{A}$ is a message containing all parameters as before except $K_{B}: \Pi_{A}=(L$, $\left.\mathrm{H}(\mathrm{N}), \mathrm{V}_{\mathrm{A}}, \Delta, \mathrm{K}_{\mathrm{A}}\right)$; similarly, $\Pi_{\mathrm{B}}=\left(\mathrm{L}, \mathrm{H}(\mathrm{N}), \mathrm{V}_{\mathrm{B}}, \Delta, \mathrm{K}_{\mathrm{B}}\right)$. Further, TTP should not have used $\mathrm{K}_{\mathrm{A}}$ and $\mathrm{K}_{\mathrm{B}}$ before.

3. $\Pi_{A}$ does not contain $K_{B}$ means that $P_{A}$ cannot encrypt $\mathbf{M}_{A}$ with $K_{B}$ (see Table 1). Therefore $M_{A}$ is $\left(L, A, H(N), \Phi_{A}\right)$ and $M_{B}$ is $\left(L, A, H(N), \Phi_{B}\right)$.

4. $P_{A}$ includes $K_{A}$ in its $A_{c k}(B)$ so that if $P_{B}$ has both $M_{A}$ and $A_{c k}(B)$ it can terminate normally without having to contact TTP. So, $\operatorname{Ack}_{A}(B)$ of Table 1 becomes $\left(\mathrm{L}, \mathrm{A}, \mathrm{H}(\mathrm{N}), \mathrm{H}\left(\mathrm{M}_{\mathrm{B}}\right), \mathrm{K}_{\mathrm{A}}\right)$ and $\mathrm{Ack}_{\mathrm{B}}(\mathrm{A})$ becomes $(\mathrm{L}, \mathrm{B}$, $\left.\mathrm{H}(\mathrm{N}), \mathrm{H}\left(\mathrm{M}_{\mathrm{A}}\right), \mathrm{K}_{\mathrm{B}}\right)$.

5. Finally, $\mathbf{M}_{\mathrm{TTP}}(\mathrm{A})$ by which TTP instructs $\mathrm{P}_{\mathrm{A}}$ to resolve the exchange, must contain $\mathrm{K}_{\mathrm{B}}: \mathrm{M}_{\mathrm{TTP}}(\mathrm{A})=\left(\mathrm{L}, \mathrm{TTP}, \mathrm{H}(\mathrm{N}), \mathrm{M}_{\mathrm{B}}, \mathrm{K}_{\mathrm{B}}\right)$. Also, $\mathrm{M}_{\mathrm{TTP}}(\mathrm{B})=$ (L, TTP, $\left.\mathrm{H}(\mathrm{N}), \mathrm{M}_{\mathrm{A}}, \mathrm{K}_{\mathrm{A}}\right)$.

Remark: Dispute Resolution: Non-repudiation guarantee ensures that when honest $\mathrm{P}_{\mathrm{A}}$ terminates normally, it has evidence that $\mathrm{P}_{\mathrm{B}}$ sent the item which $P_{A}$ delivered to $U_{A}$. However, if $U_{B}$ is dishonest, $U_{A}$ might find $I_{B}$ not passing the verification test $V_{B}$ which it agreed with $U_{B}$ in the start-up phase. Consider this scenario: malicious $U_{B}$ obtains $K_{B}$ from $\Pi_{B}$, generates $M_{B}^{\prime}$ with authentic evidence of origin but with $I_{B}^{\prime} \neq I_{B}$, and transmits $M_{B}^{\prime}$ in place of $\mathbf{M}_{B}$. Since $\Pi_{A}$ does not contain $K_{B}$ (see modification 1 above), $P_{A}$ cannot check whether the $I_{B}{ }_{B}$ in the received $M^{\prime}{ }_{B}$ meets $V_{B}$ at the end of the first round itself (see figure 2). Since $M_{B}^{\prime}$ is found to have authentic evidence of origin, it will send $A_{c k}(B)$ that contains $K_{A}$, thus letting $P_{B}$ terminate normally without ever contacting TTP. Only after being delivered of $I_{B}, U_{A}$ can find out that $I_{B}{ }_{B}$ does not pass $V_{B}$ and that $U_{B}$ has been dishonest.

If $U_{B}$ is a restricted abuser, the above scenario cannot arise: $\Pi_{B}$ is tamper-proof relative to the abilities of $U_{B}$ and therefore is can contain $K_{A}$ using which $P_{B}$ encrypts $M_{B}$ in $P 1$. Since $U_{B}$ cannot obtain $K_{A}$, he cannot modify $M_{B}$ to $M_{B}^{\prime}$ and make $P_{A}$ accept $M_{B}^{\prime}$. Thus, in $P 1$, what $U_{A}$ receives is guaranteed to be the expected one.

$\mathrm{P} 1.1$ only guarantees that $\mathrm{P}_{\mathrm{A}}$ delivers to an honest $\mathrm{U}_{\mathrm{A}}$ what a malicious $\mathrm{U}_{B}$ actually sent in exchange for $\mathrm{I}_{A}$, not necessarily what $\mathrm{U}_{B}$ has pledged to send. The TTP can guarantee fairness to $U_{A}$ in such a scenario, by contacting the trusted agent $T A_{B}$ employed to generate $V_{B}$ which $U_{A}$ accepted. This corresponds to a weaker form of fairness enforcement in the hierarchy of [9]: fairness can only be guaranteed with the help of a trusted agent outside the fair-exchange system (figure 1) and without any cooperation from $U_{B}$. The issue of post-exchange dispute resolution is discussed and addressed in $[6,7]$ for malicious abusers. The protocols of [6, 7] make use of inverse and compatible keys to verify an encrypted item without decrypting it. Using these special types of encryption keys, P1.1 can also be modified to ensure that an honest user receives in a normal termination only the item he bargained for. The modification is simple and shown in [2]. It preserves the 
2-round structure which is provably optimal [5]. The protocol of [6], like P1.1, keeps the TTP off-line but requires 4 rounds when both users are honest.

\subsection{Restricted and Malicious Abusers, Asynchronous Communication (P1A and P1.1A)}

Protocols P1 and P1.1 are not appropriate for the asynchronous model. This is not surprising as [5] shows that, in the non-fault-tolerant case, there cannot be an asynchronous protocol that works with a TTP that is off-line and state-relinquishing, and goes on to present a protocol with an off-line and state-keeping TTP. We here present protocols P1 A and P1.1A with an on-line and state-relinquishing TTP, by deriving them from P1 and P1.1 respectively. The derivation is simple: in round 2 (see figure 2), $\mathbf{P}_{\mathbf{X}}$ sends $\operatorname{Ack}_{X}(Y)$ to TTP, not to $P_{Y}$; it then waits for either $M_{T T P}(X)$ or $\operatorname{Abort}_{T T P}(X)$.

In the start-up phase, TTP should obtain round trip time (rtt) measurements with each $\mathbf{P}_{\mathrm{X}}$ (as $\mathbf{r t t}_{\mathrm{A}}$ and $\mathrm{rtt}_{\mathrm{B}}$ ) and estimate $2 \Delta$ $\operatorname{maximum}\left\{\mathrm{rtt}_{\mathrm{A}}, \mathrm{rtt}_{\mathrm{B}}, \mathrm{rtt}_{\mathrm{AB}}, \mathrm{rtt}_{\mathrm{BA}}\right\}$.

TTP:

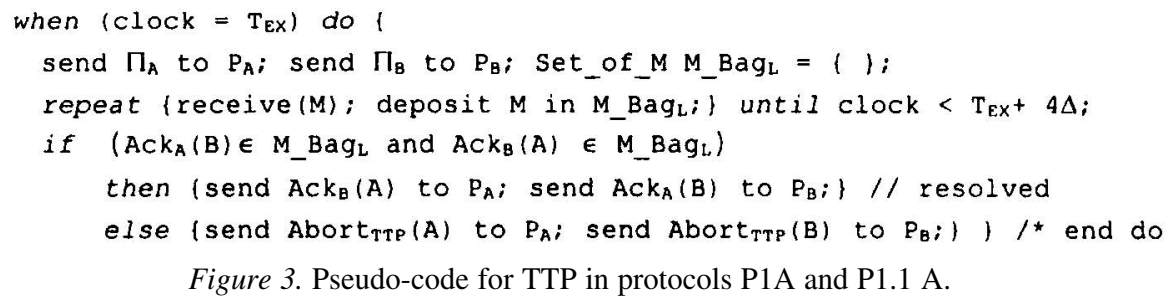

If honest users re-execute the protocol for a given exchange after every exceptional termination, non-triviality is guaranteed if there exists an execution in which the message transfer delays between $\mathbf{P}_{\mathrm{A}}, \mathrm{P}_{\mathrm{B}}$ and TTP do not exceed the $\Delta$ determined by TTP at the start of the exchange phase; i.e., if message transfer delays do not increase during an execution.

\section{CRASH-TOLERANT PROTOCOLS}

\subsection{Restricted Abuser, Synchronous Communication (P2)}

Two extensions are necessary for protocol P1 to be crash tolerant. Pessimistic (synchronous) checkpointing: $\mathrm{P}_{\mathrm{X}}$ of an honest user logs every received message and check-points its state before the received message is processed. Thus, in fig. 2, an honest $\mathbf{P}_{\mathbf{X}}$ must receive and log the messages of the incoming channels before it can send out a message. State-keeping TTP: 
in step T4, the TTP does not terminate the execution, but continues to operate depending on the value of outcome: if outcome is unknown, the TTP executes steps $\mathrm{T} 1-\mathrm{T} 3$ after a period of $4 \Delta$ time; otherwise, it responds to a message from $\mathrm{P}_{\mathrm{X}}$ with $\mathrm{M}_{\mathrm{TTP}}(\mathrm{X})$ or $\operatorname{Abort}_{\mathrm{TTP}}(\mathrm{X})$, if outcome is resolved or aborted respectively. This modification permits a recovered $\mathbf{P}_{\mathbf{X}}$ to terminate. Note that when TTP sets outcome to resolved or aborted, it is irreversible.

\section{Impossibility of making P1 Crash-tolerant}

Even with the use of pessimistic logging and state-keeping TTP, P1 cannot become crash-tolerant even if the dishonest user is a restricted abuser. Consider the following scenario. Let $N_{A}$ crash during the second round shown in figure 2 and before $P_{A}$ receives $\operatorname{Ack}_{B}(A)$; that is, $P_{A}$ has $\operatorname{logged} I_{A}$, $\Pi_{A}$, sending of $M_{A}$, and $M_{B}$. Let dishonest $U_{B}$ block all messages $P_{A}$ sent to $P_{B}$ but retains a copy of these incoming messages. $P_{B}$, having received no message from $P_{A}$ within $2 \Delta$ time, will send $R_{e q}$ for which TTP will respond by setting outcome $=$ aborted and sending $A$ bort ${ }_{\mathrm{TTP}}(\mathrm{B})$. Say $\mathrm{U}_{\mathrm{B}}$ blocks Abort ${ }_{\mathrm{TTP}}(\mathrm{B})$ as well and crashes his node. The recovered $\mathbf{P}_{\mathrm{A}}$ will find itself in having received only $\mathbf{M}_{B}$ and will send $\operatorname{Res}_{A}$ which, say, reaches TTP after outcome was set to aborted; TTP's response will be to send Abort TTP $(\mathrm{A})$ to $P_{A}$. Let $U_{B}$ re-boot $N_{B}$, delete $\Pi_{B}$ from stable-store, and adjust the clock to make it appear as if the exchange phase is being executed for the first time. He replays the arrival of $\Pi_{B}$ and of the blocked messages of $P_{A}$ exactly at those instances when they arrived during the first execution. Since $P_{B}$ 'receives' both $\mathrm{M}_{\mathrm{A}}$ and $\mathrm{Ack}_{\mathrm{A}}(\mathrm{B})$, it delivers $\mathrm{I}_{\mathrm{A}}$ to $\mathrm{U}_{\mathrm{B}}$.

\section{Outline of Protocol P2}

At the core of P1's inability to be crash tolerant is the fact that having both MA and $\operatorname{Ack}_{A}(B)$ is a sufficient condition for $P_{B}$ to deliver $I_{A}$ without consulting TTP at all. To remedy this, we need to add one more round to protocol $\mathrm{P} 1$ which $\mathrm{P}_{\mathrm{X}}$ executes after having executed the first two rounds (concurrently) and check-pointed its state. In the third round, shown in Figure $4, \mathbf{P}_{\mathbf{A}}$ sends a second acknowledgement $\mathbf{A c k}_{\mathbf{A}}{ }_{\mathbf{A}}(\mathbf{B})$ to $\mathbf{P}_{\mathbf{B}}$ indicating the reception of $\operatorname{Ack}_{\mathrm{B}}(\mathrm{A})$ and $\mathrm{M}_{\mathrm{B}}$.
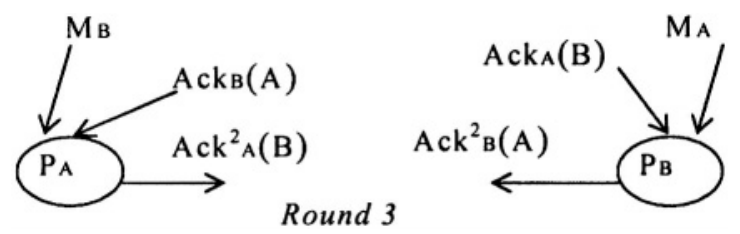

Figure 4. The additional message round in P2. 
If $P_{A}$ does not receive $\operatorname{Ack}_{B}{ }_{B}(A)$ within $2 \Delta$ time after it has sent $\operatorname{Ack}^{2}{ }_{A}(B), P_{A}$ appeals to TTP by sending First_Acks $A_{A}$ that contains both $\operatorname{Ack}_{A}(B)$ and $A_{A c k}(A)$. TTP, after receiving First_Acks $A$, sends Ack $^{2}{ }_{T T P}(A)$ to $\mathbf{P}_{\mathbf{A}}$ only if it has not earlier received $\mathbf{R e q}_{\mathbf{A}}$ (which would have resulted in sending $\mathbf{A}$ bort ${ }_{r T P}(B)$ to $\left.P_{B}\right)$. Thus, the condition for normal termination of $P_{A}$ is: $\operatorname{received}\left(\mathrm{Ack}_{2 \mathrm{~A}}^{2}(\mathrm{~A})\right)$, where $Z_{A} \in\{B, T T P\}$. By symmetry, the condition for normal termination of $P_{B}$ is: received $\left(\mathrm{Ack}_{\mathrm{ZB}}^{2}(\mathrm{~B})\right)$, where $Z_{B} \in\{A$, TTP $\}$. Three messages used in addition to those used in P1 are shown in Table 2.

\begin{tabular}{|c|c|}
\hline $\begin{array}{l}\operatorname{Ack}^{2}{ }_{\mathrm{A}}(\mathrm{B})=(\mathrm{L}, \quad \mathrm{A}, \quad \mathrm{H}(\mathrm{N}), \\
\left.\mathrm{H}\left(\mathrm{Ack}_{\mathrm{B}}(\mathrm{A})\right), M y_{-} a c k^{2}\right) ; \mathrm{A} \rightarrow \mathrm{B}\end{array}$ & $\begin{array}{l}\operatorname{Ack}^{2}{ }_{\mathrm{B}}(\mathrm{A})=(\mathrm{L}, \quad \mathrm{B}, \quad \mathrm{H}(\mathrm{N}), \\
\left.\mathrm{H}\left(\mathrm{Ack}_{\mathrm{A}}(\mathrm{B})\right), M y_{-} a c k^{2}\right) ; \mathrm{B} \rightarrow \mathrm{A}\end{array}$ \\
\hline $\begin{array}{l}\text { First_Acks }{ }_{A}=(L, \quad A, \quad H(N), \\
\left.\text { Ack }_{A}(B), A k_{B}(A)\right) ; A \rightarrow \text { TTP }\end{array}$ & $\begin{array}{l}\text { First_Acks }{ }_{B}=(\mathrm{L}, \quad \mathrm{B}, \quad \mathrm{H}(\mathrm{N}), \\
\left.\text { Ack }_{\mathrm{B}}(\mathrm{A}), \mathrm{Ack}_{\mathrm{A}}(\mathrm{B})\right) ; \mathrm{B} \rightarrow \text { TTP }\end{array}$ \\
\hline $\begin{array}{l}\mathrm{Ack}_{\mathrm{TTP}}^{2}(\mathrm{~A})=(\mathrm{L}, \quad \mathrm{TTP}, \quad \mathrm{H}(\mathrm{N}), \\
\left.\mathrm{H}\left(\mathrm{Ack}_{\mathrm{A}}(\mathrm{B})\right), M y_{-} a c k^{2}\right) ; \mathrm{TTP} \rightarrow \mathrm{A}\end{array}$ & $\begin{array}{l}\mathrm{Ack}^{2}{ }_{\mathrm{TTP}}(\mathrm{B})=(\mathrm{L}, \mathrm{TTP}, \\
\left.\mathrm{H}\left(\mathrm{Ack}_{\mathrm{B}}(\mathrm{A})\right), M y_{-} a c k^{2}\right) ; \\
\rightarrow \mathrm{B})\end{array}$ \\
\hline
\end{tabular}

Table 2. Description of additional messages used in $\mathrm{P} 2$.

\subsection{Malicious Abuser, Synchronous Communication (P2.1)}

Protocol P2.1 is the same as P2, except that some of the messages used need to be changed:

1. $\Pi_{A}, \mathbf{M}_{A}, \mathbf{M}_{\mathrm{TTP}}(\mathrm{A}), \Pi_{B}, \mathbf{M}_{B}$, and $\mathbf{M}_{\mathrm{TTP}}(\mathrm{B})$ of Table 1 change as mentioned in Section 3.2. $\operatorname{Ack}_{\mathbf{A}}(\mathbf{B})$ and $\operatorname{Ack}_{\mathbf{B}}(\mathbf{A})$ remain exactly as shown in Table 1.

2. In Table 2, My_ack $=\mathrm{K}_{\mathrm{B}}$ in $\operatorname{Ack}^{2}{ }_{\mathrm{B}}(\mathrm{A})$ and in $\mathrm{Ack}_{\mathrm{TTP}}{ }_{\mathrm{P}}(\mathrm{A})$; similarly, $M y_{-} a c k=\mathrm{K}_{\mathrm{A}}$ in $\overline{A c k}_{\mathrm{A}}{ }_{\mathrm{A}}(\mathrm{B})$ and $\mathrm{Ack}^{2}{ }_{\mathrm{TTP}}(\mathrm{B})$.

Remark: Simple Extension to Asynchronous Communication model. P2 and P2.1 work correctly in the asynchronous model if TTP computes $\Delta$ during the start-up phase as described for P1 A and P1.1A.

\subsection{Malicious and Restricted Abusers, Asynchronous Communication, on-line TTP (P2A and P2.1A)}

These protocols are obtained by making $\mathrm{P} 1 \mathrm{~A}$ and P1.1A crash-tolerant by having process $\mathbf{P}_{\mathbf{X}}$ pessimistically checkpoint its state (as in $\mathrm{P} 2$ and $\mathrm{P} 2.1$ ) before processing a received message and by making TTP state-keeping. The 
latter is done as follows: for any message received from $P_{X}$ after $T_{E X}+4 \Delta$, TTP sends $\operatorname{Ack}_{Y}(X)$ to $P_{X}$ if both $A_{c k}(B)$ and $\operatorname{Ack}_{B}(A)$ have been received before $T_{E X}+4 \Delta$; otherwise it sends Abort TTP $_{\text {TX }}(X)$.

\section{SUMMARY AND DISCUSSIONS}

Table 3 summarises the protocols' characteristics using the following abbreviations:

- a dishonest user (DU) can be either a restricted abuser (RA) or a malicious abuser (MA),

- communication delay model (C) can be synchronous (S) or asynchronous (As),

- faults tolerated (FT) can be none (No) or crash (Cr), and

- the TTP can be either off-line (Off) or on-line (On), and either staterelinquishing $(\mathrm{Sr})$ or state-keeping $(\mathrm{Sk})$.

\begin{tabular}{|l|l|l|l|l|}
\hline Protocol & DU & C & FT & TTP \\
\hline P1 & RA & S & No & Off/Sr \\
\hline P1.1 & MA & S & No & Off/Sr \\
\hline P1A(P1.1A) & RA(MA) & As & No & On/Sr \\
\hline P2 & RA & S/As & Cr & Off/Sk \\
\hline P2.1 & MA & S/As & Cr & Off/Sk \\
\hline P2A(P2.1A) & RA(MA) & As & Cr & On/Sk \\
\hline
\end{tabular}

Table 3. Characteristics of the Protocols Developed.

On-line TTP: state-relinquishing vs. state-keeping. Because of its simplicity, a non-fault-tolerant protocol with an on-line TTP is likely in practice, and a need may later arise to enhance the protocol to be crashtolerant. Can an online-TTP protocol be made crash-tolerant simply through message logging, regardless of whether the delay model is synchronous or asynchronous? The answer depends on whether the non-fault-tolerant protocol keeps the TTP state-relinquishing or state-keeping. Our protocols P1A and P1.1A suggest that it is possible to design online-TTP protocols that keep the TTP state-relinquishing even with asynchronous delays. In making them crash-tolerant, we have to make the (on-line) TTP a statekeeping one (see P2A and P2.1A in Table 3). Thus, the answer to the above question is yes, provided the issue of state-relinquishing $v s$. state-keeping TTP is given due consideration. 
Optimising the cost of message logging. The work by [4] proposes a logging-based approach to achieving crash-tolerant fair-exchange. It defines a point of no return for a user process, and claims that if the process would synchronously $\log$ all received messages before entering this point, then logging of other received messages can be done asynchronously. Their definition of point of no return suggests that there is only one such point for each process (Section 3.2 of [4]). We remark here that our protocol P2.1 without check-pointing and message logging is identical to the time-optimal protocol (scheme 3) of [5] which is asynchronous and non-fault-tolerant. Applying the approach of [4] to make this protocol crash-tolerant does not work: the point of no return for honest $\mathbf{P}_{\mathbf{A}}$ turns out to be its sending $\mathbf{M}_{\mathrm{A}}$ to $\mathbf{P}_{\mathbf{B}}$ (see figure 2, round 1); suppose that $\mathbf{N}_{A}$ crashes in round 3 (see Fig. 4) after $\mathbf{P}_{\mathrm{A}}$ has sent $\mathbf{A c k}^{2}(\mathbf{B})$ but before $\mathbf{A c k}_{B}(\mathrm{~A})$ and $\mathbf{M}_{\mathbf{B}}$ were to be logged by the asynchronous logging scheme; note that $\operatorname{Ack}_{A}{ }_{A}(B)$ contains $K_{A}$ (see Section 4.2). Honest $U_{A}$ suffers loss of fairness, even if $U_{B}$ does not misbehave; to avoid loss of fairness, $\mathbf{P}_{\mathbf{A}}$ must also log all received messages before sending $\mathrm{Ack}_{\mathrm{A}}{ }_{\mathrm{A}}(\mathrm{B})$. It appears that some protocols can have multiple points at which pessimistic logging is essential. Given that the number of messages received by a user process in a given execution is small, attempts to minimise the overhead of logging may not be worth the effort after all; further, every message received (and logged) may be useful later in any dispute resolution.

\section{Acknowledgements}

This work is funded by the UK EPSRC grant GR/N35953/01 (Information Co-ordination and Sharing in Virtual Enterprises) and by the European Union Project IST-2001-34069 TAPAS (Trusted and QoS-Aware Provision of Application Services). Our thanks are due also to the anonymous reviewers of the $17^{\text {th }}$ IFIP Conference on Database and Applications Security.

\section{REFERENCES}

[1] N. Asokan, M. Schunter and M. Waidner. Asynchronous protocols for optimistic fair exchange. IEEE Symposium on Research in Security and Privacy, pp. 86-99, 1998.

[2] P. Ezhilchelvan and S. Shrivastava. Systematic Development of a Family of Fair Exchange Protocols (Full Paper). Technical Report CS-TR-820, School of Computing Science, University of Newcastle. December 2003.

[3] M.J. Fischer, N.A. Lynch, and M.S. Paterson, "Impossibility of Distributed Consensus with one faulty Process," Journal of the ACM, Vol. 32, No. 2, pp. 374-382, April 1985. 
[4] P. Liu, P. Ning and S. Jajodia. Avoiding Loss of Fairness Owing to Process Crashes in Fair Data Exchange Protocols. Inernational Conference on Dependable Systems and Network. pp. 631-40, June 2000.

[5] B. Pfitzmann, M. Schunter and M. Waidner. Optimal Efficiency of Optimistic Contract Signing. Proc. ACM Symp. on Principles of Distributed Computing. New York, 1998.

[6] I. Ray and I. Ray. An Optimistic Fair Exchange E-commerce Protocol with Automated Dispute Resolution. EC-Web 2000, pp. 84-93.

[7] I. Ray, I. Ray, N. Narasimhamurthy: A Fair-exchange E-commerce Protocol with Automated Dispute Resolution. DBSec 2000, pp. 27-38.

[8] Trusted Computing Platform Alliance, http://www.trustedpc.org.

[9] H. Vogt, H. Pagnia, and F. C. Gartner. Modular fair exchange protocols for electronic commerce. 15th Annual Computer Security Applications Conference, pp. 3-11, Dec. 1999.

[10] H. Vogt, H. Pagnia, and F. C. Gartner. Using Smart cards for Fair-Exchange. WELCOM 2001, LNCS 2232, Springer, pp. 101-113, 2001.

[11] J. Zhou and D. Gollmann. Evidence and non-repudiation. Journal of Network and Computer Applications, 20(3):267-281, July 1997. 